\title{
Photoemission Studies of Si Quantum Dots with Ge Core: Dots formation, Intermixing at Si-clad/Ge-core interface and Quantum Confinement Effect
}

\author{
Yudi Darma ${ }^{1}$ \\ ${ }^{1}$ Quantum Semiconductor and Devices Laboratory, \\ Study Program of Physics, Bandung Institute of Technology, \\ Ganesha 10 Bandung Indonesia 40132 \\ yudi@fi.itb.ac.id
}

\begin{abstract}
Spherical Si nanocrystallites with Ge core ( $20 \mathrm{~nm}$ in average dot diameter) have been prepared by controlling selective growth conditions of lowpressure chemical vapor deposition (LPCVD) on ultrathin $\mathrm{SiO}_{2}$ using alternately pure $\mathrm{SiH}_{4}$ and $5 \% \mathrm{GeH}_{4}$ diluted with He. XPS results confirm the highly selective growth of $\mathrm{Ge}$ on the pregrown $\mathrm{Si}$ dots and subsequently complete coverage by $\mathrm{Si}$ selective growth on $\mathrm{Ge} / \mathrm{Si}$ dots. Compositional mixing and the crystallinity of Si dots with Ge core as a function of annealing temperature in the range of $550-800^{\circ} \mathrm{C}$ has been evaluated by XPS analysis and confirms the diffusion of $\mathrm{Ge}$ atoms from $\mathrm{Ge}$ core towards the Si clad accompanied by formation of $\mathrm{GeO}_{\mathrm{x}}$ at the $\mathrm{Si}$ clad surface. The first subband energy at the valence band of Si dot with Ge core has been measured as an energy shift at the top of the valence band density of state using XPS. The systematic shift of the valence band maximum towards higher binding energy with progressive deposition in the dot formation indicate the charging effect of dots and $\mathrm{SiO}_{2}$ layer by photoemission during measurements.
\end{abstract}

Keywords: charging effect; compositional mixing; Ge core; LPCVD; photoemission; Si dot; Thermal stability.

\section{Introduction}

Nanometer size dots with a Si/Ge stacking structure have attracted extensive interest because of their unique properties associated with carrier confinement [1]. Si/Ge nanoislands becomes an alternative candidate to realize novel functional devices instead of pure nanocrystalline $\mathrm{Si}$ on ultrathin $\mathrm{SiO}_{2}$ in which is in progress to develop some functional devices such as resonant tunneling devices [2], single electron transistors [3] and quantum dot memories [4]. So far, lateral and/or vertical ordering of Ge islands on patterned of Si [5] and the selective overgrowth of $\mathrm{Ge}$ on $\mathrm{Si}$ islands on $\mathrm{Si}(001)$ windows have been reported [6]. These experimental results indicate that the strain relaxation by local elastic deformation plays a role in the island formation and the ordering of islands leads to good uniformity in size. Furthermore, the thermal stability of

Received June $6^{\text {th }}, 2006$, Revised April 19 ${ }^{\text {th }}, 2007$, Accepted for publication April $19^{\text {th }}, 2007$. 
SiGe/Si and Ge/Si interfaces, has so far been studied in $\mathrm{Si}_{1-\mathrm{x}} \mathrm{Ge}_{\mathrm{x}} / \mathrm{Si}$ superlattices [7], SiGe nano-islands embedded in Si [8] and Ge nano-islands on Si [9]. It has been reported that the interdiffusion coefficient of the nano-islands was much higher than that of layered structures and also atomic interdiffusion can decrease the strain energy.

In our previous work, we have demonstrated the self-assembling of nanometer Si dots on $\mathrm{SiO}_{2}$ by controlling the early stages of LPCVD of monosilane $\left(\mathrm{SiH}_{4}\right)$ and succeeded in the positioning and spatial arrangement of Si dots on ultrathin $\mathrm{SiO}_{2}$ with the control of nucleation or reactive sites such as surface OH-bonds [10]. We have also confirmed unique multiple-step charging characteristics of Si quantum dots at room temperature [11].

For the potential modulation in Si dots and the enhancement of carrier confinement, we extend our research to the formation of Si dots with Ge core by alternately controlling the selective growth conditions in LPCVD using pure $\mathrm{SiH}_{4}$ and $\mathrm{GeH}_{4}$ [12]. To gain a better understanding of compositional intermixing at $\mathrm{Si}$ clad and $\mathrm{Ge}$ core interface, the isochronal annealing experiments were carried out as well.

In this paper, we focus the photoemission measurements to study the Si dot with Ge core formation, compositional intermixing at the Si dots with Ge core interface as a function of annealing temperature accompanied by quantum confinement effect of Ge core cladding by Si nanocrystalline.

\section{Experimental}

Hemispherical single-crystalline Si dots were first grown on thermally grown $\mathrm{SiO}_{2}$ on $\mathrm{Si}(100)$ at $560^{\circ} \mathrm{C}$ from the thermal decomposition of pure $\mathrm{SiH}_{4}$ by maintaining the gas pressure at 0.5Torr. Ge deposition was performed selectively on pregrown $\mathrm{Si}$ dots at $400^{\circ} \mathrm{C}$ for 3 min using $5 \% \mathrm{GeH}_{4}$ diluted with $\mathrm{He}$ and subsequently followed by the $\mathrm{Si}$ selectively deposition on the dots at $540^{\circ} \mathrm{C}$ under a $\mathrm{SiH}_{4}$ pressure of $0.02 \mathrm{Torr}$. To minimize the compositional mixing during this preparation process, after each deposition step, CVD chamber was completely purged with dry $\mathrm{N}_{2}$ and evacuated down to $\sim 10-7$ Torr. Experiment flow is presented in schematic viewgraph in Figure1. Some of the samples were annealed isochronally for $5 \mathrm{~min}$ each in temperature range of 550$800^{\circ} \mathrm{C}$ in $\mathrm{N}_{2}$ ambient. To confirm the selective growth of Ge and $\mathrm{Si}$ cap in the dot formation and the structural change during thermal annealing, highresolution X-Ray photoelectron spectroscopy (XPS) measurements were carried out using monochromatized AlK $\alpha$ radiation (1486.6 eV). O1s, C1s, Si2p and Ge3d core spectra and subsequently valence spectra were taken systematically with an energy step of $50 \mathrm{meV}$ at an electron pass energy of $20 \mathrm{eV}$. Additionally, 
XPS measurement with take-off angle of $15^{\circ}$ was chosen for surface sensitive measurement. The formation of Si dot with Ge core has been investigated systematically using atomic force microscopy (AFM), high-resolution transmission electron microscopy (HR-TEM) and Raman spectroscopy in ref. $12[12]$.
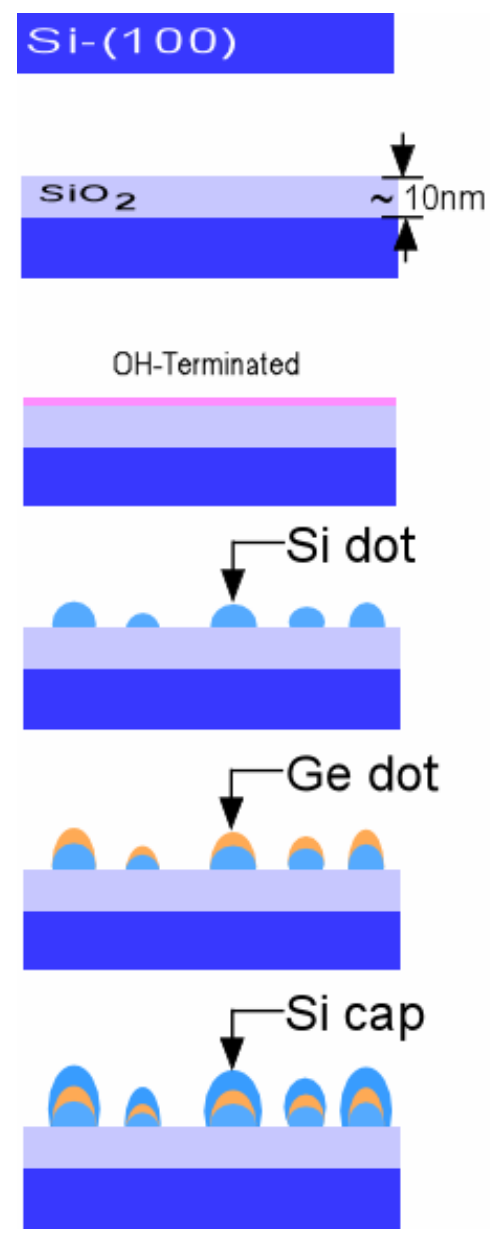

Figure 1 Schematic viewgraph of quantum dot fabrication.

\section{$3 \quad$ Results and Discussion}

Highly-selective deposition of Ge and subsequent Si on the dots was confirmed from the XPS analysis of native oxides on the dot surfaces formed during air exposure as indicated in Figure 2. For the sample of pregrown Si dots on $\mathrm{SiO}_{2}$, an intense peak due to mainly $\mathrm{Si}^{4+}$ in the underlying $\mathrm{SiO}_{2}$ layer and partly the 
native oxide on top surface of Si dots is observed at $~ 104 \mathrm{eV}$ accompanied with weak signals peaked at $\sim 100 \mathrm{eV}$ due to $\mathrm{Si}^{0+}$ in the $\mathrm{Si}$ dots. In contrast, little $\mathrm{Si}^{0+}$ signals are detectable for as-prepared thick $\mathrm{SiO}_{2}$ just before $\mathrm{Si}$ dots formation (data is not shown). By Ge deposition, Si2 $\mathrm{p}^{4+}$ signals are markedly weaken compared with a slightly decrease in $\mathrm{Si} 2 \mathrm{p}^{0+}$ signals. This is interpreted in term that Ge coverage of Si dot surface results in the efficient suppression of surface oxidation of Si dots during air exposure. Almost complete coverage of the pregrown Si surface with Ge was also verified from the following experimental result, where the XPS spectra of samples exposed to the clean room air for 5 and 45 min after Ge deposition are compared with the spectra after dipping the $45 \mathrm{~min}$ air-exposed sample into $0.1 \%$ HF solutions for $60 \mathrm{~s}$. After the HF treatment, the $\mathrm{Ge}^{4+}$ component almost completely disappears while no changing of $\mathrm{Si}^{4+}$ intensity is detectable, and also $\mathrm{Si}^{0+}$ signal intensity is recovered to the same intensity as the $5 \mathrm{~min}$ air-exposed sample. The result indicates that only Ge surface oxide formed by $45 \mathrm{~min}$ air exposure is removed by dilute HF treatment, that is, the pregrown $\mathrm{Si}$ surface is passivated with Ge. When Ge deposition was followed by $\mathrm{Si}$ deposition for 30s, the chemically-shifted Ge signals from surface GeOx become hardly observed. This indicates that Ge surface is almost perfectly passivated with $\mathrm{Si}$. From separated experiments, we have also confirmed that the deposition of Ge on both as-grown and HF-treated $\mathrm{SiO}_{2}$ form $5 \% \mathrm{GeH}_{4}$ dilute with He was hardly observable in the temperature range of $300-520^{\circ} \mathrm{C}$ under the gas pressure of 0.02 - 0.5Torr, which is presumably caused by a surface reaction product such as Ge monoxide is volatile (shown in Figure 2). Furthermore, from Figure 4, Ge coverage of pregrown $\mathrm{Si}$ dot with 1min deposition clearly indicate that the efficient suppression of native oxidation on Si dot surface. Further, pregrown Si dot became deeper from the surface with progressive Ge depositions. In Ge3d spectra, chemically shift due to $\mathrm{GeO}_{\mathrm{x}}$ units is observed that mainly due to the surface oxidation after air exposure, and obviously $\mathrm{Ge}^{\mathrm{O}+}$ signals is increasing markedly with increasing Ge deposition time. Also in the early stages of Si cap formation as presented in Figure 5, In the early stages of Si cap formation, the chemically-shifted signals due to $\mathrm{GeO}_{\mathrm{x}}$ is still observable even for the case of 20s deposition in which the Si2p spectrum being almost identical to that of 30s deposition was taken at the photoelectron take-off angle of $15^{\circ}$, where the photoelectron escape depth is as small as $\sim 0.8 \mathrm{~nm}$. Considering no significant change in $\mathrm{Si} 2 \mathrm{p}^{0+}$ spectra, such a oxide component can be interpreted as a result of non-uniform formation of the Si cap or the fluctuation of Si covered rather than the intermixing between $\mathrm{Ge}$ and $\mathrm{Si}$. If the change in $\mathrm{Ge}^{0+}$ signals is due to the chemical-shift by Si-Ge bonding, the chemical-shifted component to the opposite binding energy side should be observed in $\mathrm{Si}^{0+}$ signals. In addition, in the 2nd Si-LPCVD, spontaneous nucleation rate on $\mathrm{SiO}_{2}$ was suppressed efficiently under the gas pressure as low as 0.02 Torr, in which the thermal desociation of small nuclei is promoted. 

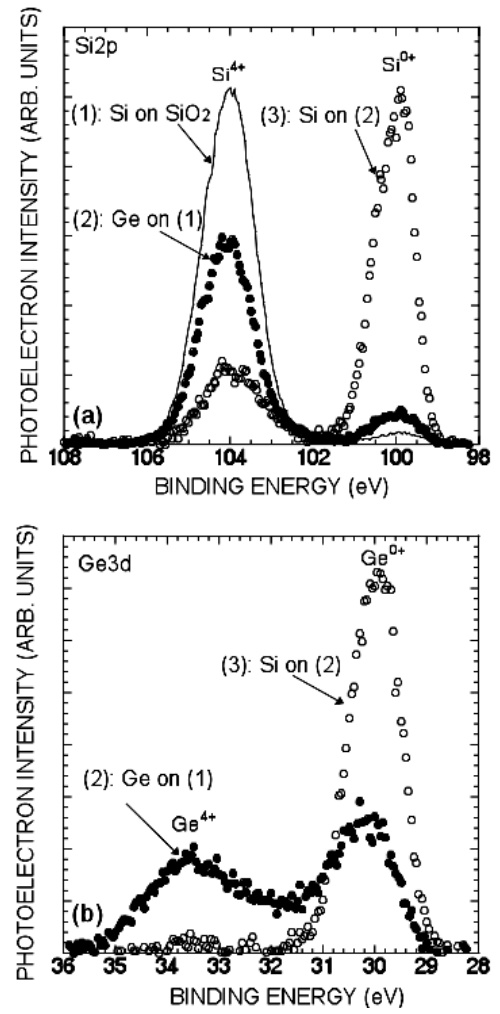

Figure 2 XPS spectra of Si2p (a) and Ge3d (b) . Si-dot pregrown on $\mathrm{SiO}_{2}$ (the sample (1), Ge deposition on sample (1) (the sample (2) and Si deposition on sample (2) (sample (3), The samples were exposed to the clean room air for $\sim 5$ $\min$ at room temperature. The photoelectron take-off angle was set at $15^{\circ}$

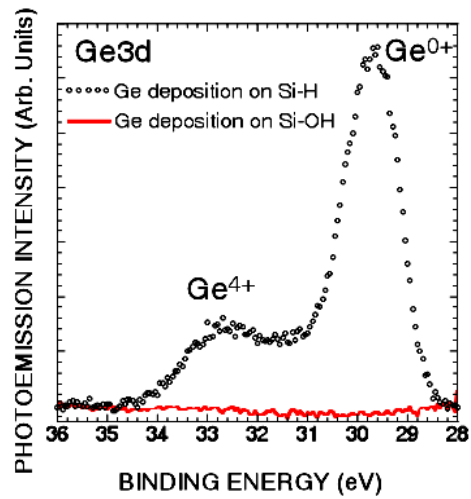

Figure 3 XPS spectra of Ge3d for GeH4 chemical vapor deposition on $\mathrm{Si}$ (open circle) and $\mathrm{SiO}_{2}$ (solid line). 

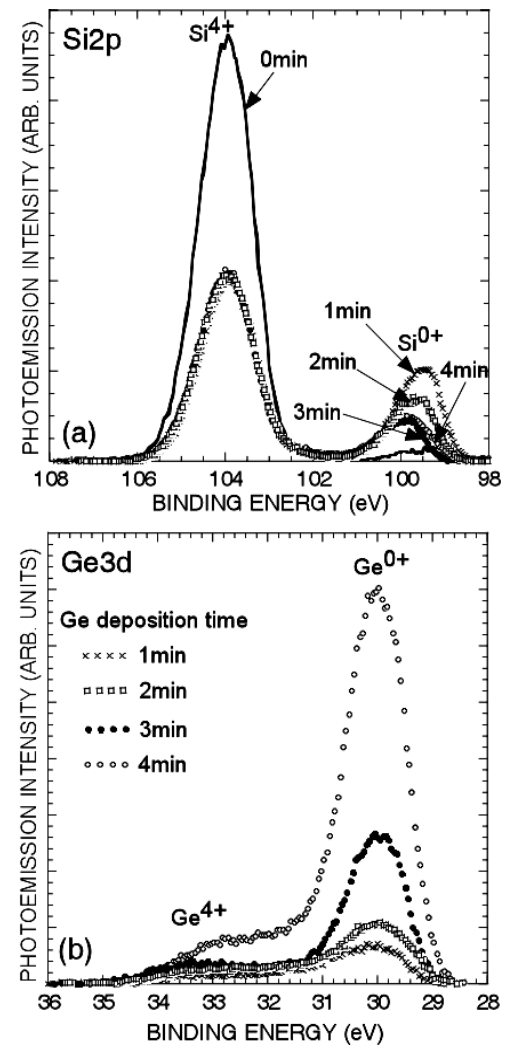

Figure $4 \mathrm{Si} 2 \mathrm{p}$ (a) and Ge3d (b) spectra of the samples exposed to clean-room air for $5 \mathrm{~min}$ after the Ge deposition on $\mathrm{Si}$ dots $/ \mathrm{SiO}_{2}$. Ge deposition time was change from 1-4 min.

The compositional intermixing between the Si clad and the Ge core was confirmed by the evolution of XPS spectra with annealing experiment at temperature range of $550-800^{\circ} \mathrm{C}$. Figure 6 shows the Si2p and Ge3d spectra for annealed samples as a function of annealing temperature. With increasing annealing temperature, $\mathrm{Si}^{0+}$ signals originating from $\mathrm{Si}$ clad decrease gradually, and $\mathrm{Si}^{4+}$ signals from the underlying $\mathrm{SiO}_{2}$ and surface oxide on the dot surface and $\mathrm{Ge}^{0+}$ signals from $\mathrm{Ge}$ core increase slightly. The result indicates that, although Si clad surface was oxidized during annealing after air exposure, Ge atoms diffuse toward the dot surface, that is, compositional mixing at $\mathrm{Si} / \mathrm{Ge}$ interface. Notice that chemically-shifted Ge3d signals assigned to $\mathrm{Ge}^{4+}$ in oxide increase until it becomes its maximum at an annealing temperature around $650^{\circ} \mathrm{C}$. This indicates that thermal desorption of $\mathrm{GeO}_{\mathrm{x}}$ becomes significant at temperatures higher that $650^{\circ} \mathrm{C}$. 

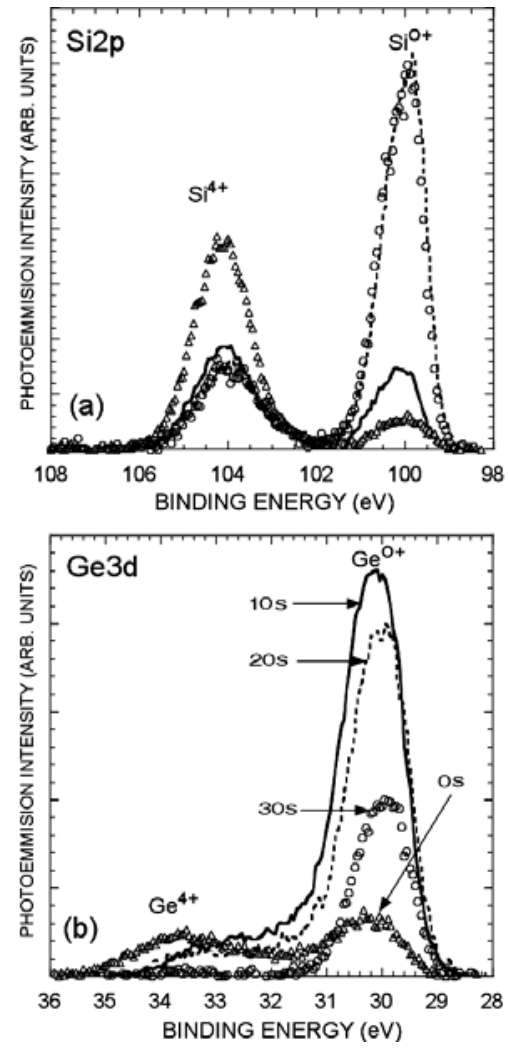

Figure $5 \mathrm{Si} 2 \mathrm{p} \mathrm{(a)}$ and Ge3d (b) spectra of the samples after the Si deposition on $\mathrm{Ge} / \mathrm{Si}$ dots $/ \mathrm{SiO}_{2}$. For the case of 10 s deposition, the signals from a bottom $\mathrm{SiO}_{2}$ layer also contribute to the chemically-shifted signals assigned to $\mathrm{Si}^{4+}$. Spectra for the sample before Si cap deposition also presented as reference.

Regarding of the valence bands spectra for each deposition step as shown in Figure 7, the valence band edge of each deposition step exhibits a shift of about $0.4 \mathrm{eV}$ to the higher binding energy. While for the pure Si dot, the shift was obtained as higher as $0.6 \mathrm{eV}$ [13]. This shift arises not only from the dot charging effect but also from the subband formation in the dot as confirmed by photoluminescence spectra that will be discussed in detail elsewhere. It should be note that the valence band spectra from 11-17 eV originate from the Si3pO2p and Si3s-O2p bonding orbitals in the oxide layer [14]. A relative shift of the valence band peak position at about $12 \mathrm{eV}$ afer Si and Ge deposition appear to be less positively charged than the valence band from oxide layer. As a matter of fact, the shift of peak position due to the non-uniform charging effect is rather small compared to the magnitude of valence band maximum shifts that could be attributed to the quantum confinement. 

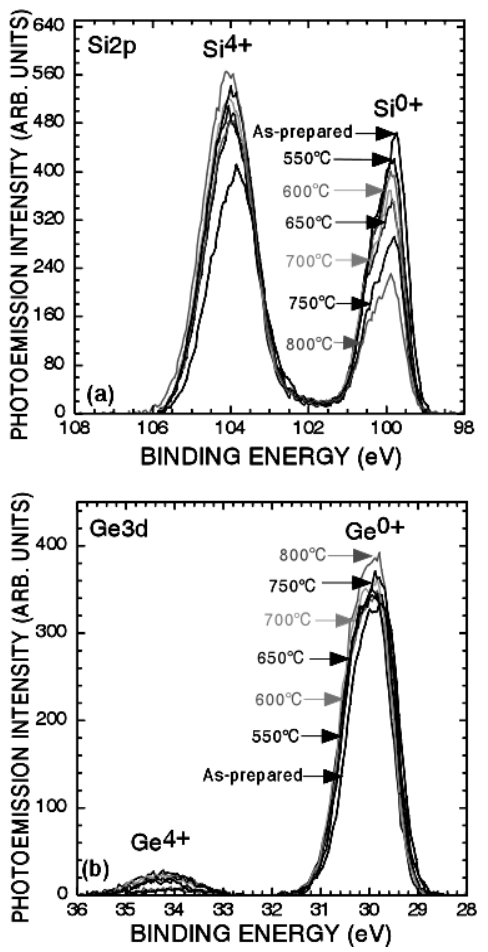

Figure 6 XPS spectra of Si2p and Ge3d for as-prepared and annealed samples at different temperatures, which were taken at photoemission take-off angle of 90o. For this experiment, a single layer of Si dots with Ge core was prepared on 10nm-thick $\mathrm{SiO}_{2} / \mathrm{Si}(100)$.

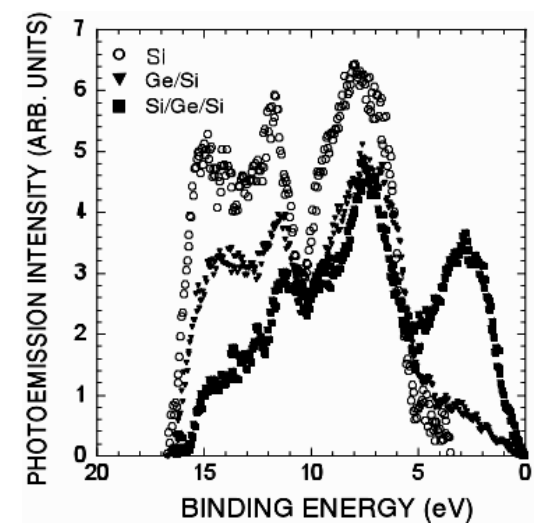

Figure 7 Valence band spectra of Si-dot pregrown on $\mathrm{SiO}_{2}$ : sample (1): circle, Ge deposition on sample (1); sample (2): triangle and Si deposition on sample (2); sample (3): square. 


\section{Conclusion}

We have studied systematically the highly selective growth of Ge on pregrown Si dots and subsequent complete coverage with a Si cap that enables us to embed a Ge core into each of spherical Si dots as confirmed from XPS spectra. For annealed samples, a gradual increase in Ge signals from metallic states with annealing higher than $650{ }^{\circ} \mathrm{C}$ confirms the diffusion of Ge atoms into the Si clad and a decrease in the chemically-shifted Ge signals originated from oxide is indicative of thermal desorption of volatile Ge oxide units. Moreover XPS analysis confirm the quantum confinement effect in nanometer Si dots with Ge core by shifting of the valence band maximum towards the higher binding energy with progressive deposition.

\section{Acknowledgements}

The author would like to thank Prof. S. Miyazaki for his useful discussion and M. Yamaoka and A. Ohta for their help in XPS measurements.

\section{References}

[1] Vescan, L., Cryst, J., Growth, 194, 173, 1998.

[2] Fukuda , M., Nakagawa , K., Miyazaki, S., Hirose, M., Appl. Phys. Lett., 70, 2291, 1997.

[3] Takahashi, Y., Fujiwara, A., Yamasaki, K., Namatsu, H., Kurihara, K., Murase, K., Abs. Int. Conf. Solid State Devices Mater, pp.176, 1998.

[4] Tiwari, S., Rana, E., Hanafi, H., Hartstein, A., Crabb, E.,E., Chan, K., Appl. Phys. Lett., 68, 1377, 1996.

[5] Kamins, T. I., and Williams, R. S., Appl. Phys. Lett., 71, 1201, 1997.

[6] Nitta, Y., Shibata, M., Fujita, K., and Ichikawa, M., Surf. Sci., 496, L7, 2002.

[7] Kamins, T.I., Medeiros-Ribeiro, G., Ohlberg, D. A. A., Williams, R. S., J. Appl. Phys., 85, 1159, 1999.

[8] Wan, J., Luo, Y. H., Jiang, Z. M., Jin, G., Liu, J.L., Wang, Kang L., Liao, X.Z., and Zou, J., J. Appl. Phys., 90(8), 4290, 2001.

[9] Schorer, R., Friess, E., Eberl, K., Abstreiter, G., Phys. Rev. B, 44, 1772, 1991.

[10] Miyazaki, S., Yamamoto, Y., Yoshida, E., Ikeda, M., and Hirose, M., Thin Solid Films, 369, 55, 2000.

[11] Kohno, A., Murakami, H., Ikeda, M., Miyazaki, S., and Hirose, M., Jpn. J. Appl. Phys., 40, L721, 2001.

[12] Darma, Y., Murakami, H., Miyazaki, S., Nanotechnology, 14, 413, 2003.

[13] Ding, S. A., Ikeda, M., Fukuda, M., Miyazaki, S., and Hirose, M., Appl. Phys. Lett., 73, 3881, 1998.

[14] Alay, J. L., and Hirose, M., J. Appl. Phys., 81, 1606, 1997. 